\title{
A NOTE ON THE NON-INTEGRABILITY OF SOME HAMILTONIAN SYSTEMS WITH A HOMOGENEOUS POTENTIAL*
}

\author{
JUAN J. MORALES-RUIZ ${ }^{\dagger}$ AND JEAN PIERRE RAMIS $\ddagger$
}

\begin{abstract}
We obtain a non-integrability result on Hamiltonian Systems with a homogeneous potential with an arbitrary number of degrees of freedom which generalizes a Yoshida's Theorem [7]. Except for the cases when the degree of homogeneity of the potential is equal to two or minus two, only a discrete set of families of these type of potentials are compatible with the complete integrability condition. We illustrate this result with two examples: the collinear problem of three bodies and a highly symmetrical family introduced by Umeno ([6]).
\end{abstract}

1. Introduction. The purpose of this note is to give a simple non-integrability criterion for complex Hamiltonian Systems with homogeneous potentials, i.e., of the type

$$
H(x, y)=T+V=\frac{1}{2}\left(y_{1}^{2}+\ldots+y_{n}^{2}\right)+V\left(x_{1}, \ldots, x_{n}\right),
$$

where $V$ is a homogeneous function of integer degree $k$. We consider this as a first non exclusively academic application in order to test the results of our previous paper [4]. In this way, we improve some Yoshida's results even for two degrees of freedom (see [7]) and we avoid the arithmetical problems related with the non-resonance assumptions in Ziglin's Theorem or its generalizations, that are exclusively based on an analysis of the monodromy group of the variational equations (see [6]).

As two concrete examples we study the collinear homogeneous problem of three particles, studied by Yoshida in [7], and the $n$-degrees of freedom system with potential

$$
V=\frac{1}{s} \sum x_{i_{1}}^{s} x_{i_{2}}^{s} \cdots x_{i_{r}}^{s}
$$

studied by Umeno $([6])$.

We note that from Kimura's Theorem (Theorem 2) and our Theorem 1, the methods of this note are completely systematic and elementary. In our opinion, that reflects the fact that the natural setting to obtain non-integrability results, using an analysis of the variational equations (along a particular integral curve), is the Differential Galois Theory.

2. Non-integrability Theorem. Consider a complex analytic symplectic manifold $M$ of dimension $2 n$ and let $X_{H}$ be a holomorphic Hamiltonian system defined on it. Let $\Gamma$ be the (abstract) connected Riemann surface corresponding to an integral curve $C$ defined by $z=z(t)$ (which is not an equilibrium point) of $X_{H}$. Then we can obtain the variational equations $(V E)$

$$
\dot{\eta}=X_{H}^{\prime}(z(t)) \eta
$$

over along $\Gamma$, and we can obtain, also, the normal variational equations ( $N V E)$ along $\Gamma$. These variational equations are given by holomorphic (resp. meromorphic) connections over $\Gamma$ and it is possible to interpret them as meromorphic linear differential equations over $\Gamma$. (For more details see [4].)

\footnotetext{
*Received March 1, 2000; revised April 6, 2001.

${ }^{\dagger}$ Departament de Matemàtica Aplicada II, Universitat Politècnica de Catalunya, Pau Gargallo 5, E-08028 Barcelona, Spain (morales@ma2.upc.es).

${ }^{\ddagger}$ Laboratoire Emile Picard, Université Paul Sabatier, 118, route de Narbonne, Toulouse, Cedex, France and Institut Universitaire de France (ramis@picard.ups-tlse.fr).
} 
Then, in the above situation, we prove in [4] the following result

THEOREM 1. Assume that there are $n$ meromorphic first integrals of $X_{H}$ in involution and independent in a neighborhood of the curve $C$ in $M$, not necessarily on $C$ itself. Then the identity component of the Galois group of the VE (resp. (NVE)) is an abelian subgroup of the symplectic group.

In particular, the $V E$ (and the $N V E$ ) are solvable in the sense of the Differential Galois Theory. To be more precise, the Picard-Vessiot extension of the VE (and of the $N V E$ ) is obtained by (a combination of) quadratures, exponential of quadratures and algebraic functions. We recall that this is the case if, and only if, the identity component of the Galois group is solvable (see, for instance $[1,3,5]$ ).

In the above theorem the differential Galois group $G$ is computed relatively to the differential field $k_{\Gamma}$ of meromorphic functions over $\Gamma$. Then it is well known that $G$ is the Zariski closure in the general linear group of the monodromy group of the variational equation. (That is $G$ the smallest complex algebraic linear group containing the monodromy group.)

In many practical situations (as in the examples of homogeneous potentials below), it is possible to add to our Riemann surface $\Gamma$ a finite number of points (corresponding in general to equilibrium points in $M$ or points at infinity) such that we get a connected compact Riemann surface $\bar{\Gamma}$ and such that the connection of the $V E$ extends as a meromorphic connection over $\bar{\Gamma}$. Then we can compute the differential Galois group $\bar{G}$ of the $V E$ relatively to the differential field $k_{\bar{\Gamma}}$ of meromorphic functions over $\bar{\Gamma}$. Then $G \subset \bar{G}$, but in general this inclusion is strict. This is unfortunate, because in general it is more easy to compute $\bar{G}$ than $G$. (Theoretically there exists an algebraic algorithm to decide if $\bar{G}$ is solvable.) Happily when the extended connection of the $V E$ over $\bar{\Gamma}$ is Fuchsian (that is when its singular points are regular singular), then we have $G=\bar{G}$. It will be the case in the present paper and therefore we will get a purely algebraic systematic method to decide if the identity component of $G$ is not abelian (in fact non solvable). (This will be based upon Kimura's result about hypergeometric differential equations.)

3. Application to homogeneous potentials. Let an $n$ degrees of freedom Hamiltonian System with Hamiltonian

$$
H(x, y)=T+V=\frac{1}{2}\left(y_{1}^{2}+\ldots+y_{n}^{2}\right)+V\left(x_{1}, \ldots, x_{n}\right),
$$

being $V$ a homogeneous function of integer degree $k$ and $2 \leq n$. For the case $n=2$, Yoshida obtained a remarkable non-integrability theorem based on Ziglin's Theorem ([7]).

As Yoshida notes, it is not possible to generalize, in a direct way, his theorem to $n>2$. Indeed, it is difficult to check the non-resonant condition of Ziglin's Theorem. He asks for a generalization of Ziglin's Theorem in order to handle these systems ([7], p. 141). With Theorem 1 it is possible to solve this problem and furthermore, even for the case $n=2$, to improve Yoshida's results.

We will follow the Yoshida arguments in order to obtain a set of hypergeometric equations as the $N V E$ along a particular solution of the Hamiltonian System $H(x, y)$ above. From the homogeneity of $V$, it is possible to get an invariant plane

$$
\begin{aligned}
& x=z(t) c, \\
& y=\dot{z}(t) c, i=1,2, \ldots, n,
\end{aligned}
$$


where $z=z(t)$ is a solution of the (scalar) hyperelliptic differential equation

$$
\dot{z}^{2}=\frac{2}{k}\left(1-z^{k}\right)
$$

(where we assume case $k \neq 0$ ), and $c=\left(c_{1}, c_{2}, \ldots c_{n}\right)$ is a solution of the equation

$$
c=V^{\prime}(c)
$$

This is our particular solution, $\Gamma$, along which we compute the $V E$ and the NVE.

The $V E$ along $\Gamma$ is given (in the temporal parametrization) by

$$
\ddot{\eta}=-z(t)^{k-2} V^{\prime \prime}(c) \eta
$$

By the symmetry of the Hessian matrix $V^{\prime \prime}(c)$, it is possible to express the $V E$ as a direct sum of second order equations

$$
\ddot{\eta}_{i}=-z(t)^{k-2} \lambda_{i} \eta_{i}, i=1,2, \ldots, n,
$$

where we preserve $\eta$ for the new variable, being $\lambda_{i}$ the eigenvalues of the matrix $V^{\prime \prime}(c)$. We will call Yoshida coefficients these eigenvalues. One of the above second order equations is the tangential variational equation, say, the equation corresponding to $\lambda_{n}=k-1$. This equation is trivialy solvable and we get as $N V E$ an equation in the variables $\xi:=\left(\eta_{1}, \ldots, \eta_{n-1}\right):=\left(\xi_{1}, \ldots, \xi_{n-1}\right)$, i.e.,

$$
\ddot{\xi}=-z(t)^{k-2} \operatorname{diag}\left(\lambda_{1}, \ldots, \lambda_{n-1}\right) \xi .
$$

Now, following Yoshida, we consider the finite branched covering map

$$
\bar{\Gamma} \rightarrow \mathbf{P}^{1},
$$

given by $t \mapsto x$, being $x=: z(t)^{k}$ (here $\bar{\Gamma}$ is the compact hyperelliptic Riemann surface of the hyperelliptic curve $w^{2}=\frac{2}{k}\left(1-z^{k}\right)$, see [4] for the notation and technical details). By the symmetries of this problem, we get as $N V E$ a system of independent hypergeometric differential equations in the new independent variable $x$

$$
x(1-x) \frac{d^{2} \xi}{d x^{2}}+\left(\frac{k-1}{k}-\frac{3 k-2}{2 k} x\right) \frac{d \xi}{d x}+\frac{\lambda_{i}}{2 k} \xi=0, \quad i=1,2, \ldots, n-1 .
$$

This system of equations is the algebraic normal variational equation $(A N V E)$. If we write $A N V E_{i}$ for the scalar second order equation corresponding to the Yoshida coefficient $\lambda_{i}$ then

$$
A N V E=A V N E_{1} \oplus A V N E_{2} \oplus \ldots \oplus A V N E_{n-1},
$$

(in fact it is a direct sum in the more intrinsic sense of linear connections, see [4]). Then it is clear that the $A N V E$ is solvable if, and only if, each one of the $A N V E_{i}$ 's is also solvable. In other words, the identity component of the Galois Group of the $A N V E$ is solvable if, and only if, each one of the identity components of the Galois Group of the $A V N E_{i}$ 's $i=1,2, \ldots, n-1$, is solvable.

Each one of the above $A N V E_{i}$ is an hypergeometric equation with three regular singular points at $x=0, x=1$ and $x=\infty$. We remark that, by a result proved in [4], the identity component of the Galois Group of the $N V E$ is the same as the identity component of the Galois Group of the ANVE. 
For the equation $A N V E_{i}$, the exponent differences at $x=0, x=\infty$ and $x=1$ are (respectively) $\hat{\lambda}=1 / k, \hat{\mu}=\sqrt{(k-2)^{2}+8 k \lambda_{i}} /(2 k)$ and $\hat{\nu}=1 / 2$. Now, we recall the theorem of Kimura ([2]) already mentioned in [4]

TheOREM 2. The identity component of the Galois Group of the hypergeometric equation is solvable if, and only if, either

(i) At least one of the four numbers $\hat{\lambda}+\hat{\mu}+\hat{\nu},-\hat{\lambda}+\hat{\mu}+\hat{\nu}, \hat{\lambda}-\hat{\mu}+\hat{\nu}, \hat{\lambda}+\hat{\mu}-\hat{\nu}$ is an odd integer, or

(ii) The numbers $\hat{\lambda}$ or $-\hat{\lambda}$, $\hat{\mu}$ or $-\hat{\mu}$ and $\hat{\nu}$ or $-\hat{\nu}$ belong (in an arbitrary order) to some of the following fifteen families

\begin{tabular}{|c|c|c|c|c|}
\hline 1 & $1 / 2+l$ & $1 / 2+m$ & arbitrary complex number & \\
\hline 2 & $1 / 2+l$ & $1 / 3+m$ & $1 / 3+q$ & \\
\hline 3 & $2 / 3+l$ & $1 / 3+m$ & $1 / 3+q$ & $l+m+q$ even \\
\hline 4 & $1 / 2+l$ & $1 / 3+m$ & $1 / 4+q$ & \\
\hline 5 & $2 / 3+l$ & $1 / 4+m$ & $1 / 4+q$ & $l+m+q$ even \\
\hline 6 & $1 / 2+l$ & $1 / 3+m$ & $1 / 5+q$ & \\
\hline 7 & $2 / 5+l$ & $1 / 3+m$ & $1 / 3+q$ & $l+m+q$ even \\
\hline 8 & $2 / 3+l$ & $1 / 5+m$ & $1 / 5+q$ & $l+m+q$ even \\
\hline 9 & $1 / 2+l$ & $2 / 5+m$ & $1 / 5+q$ & $l+m+q$ even \\
\hline 10 & $3 / 5+l$ & $1 / 3+m$ & $1 / 5+q$ & $l+m+q$ even \\
\hline 11 & $2 / 5+l$ & $2 / 5+m$ & $2 / 5+q$ & $l+m+q$ even \\
\hline 12 & $2 / 3+l$ & $1 / 3+m$ & $1 / 5+q$ & $l+m+q$ even \\
\hline 13 & $4 / 5+l$ & $1 / 5+m$ & $1 / 5+q$ & $l+m+q$ even \\
\hline 14 & $1 / 2+l$ & $2 / 5+m$ & $1 / 3+q$ & $l+m+q$ even \\
\hline 15 & $3 / 5+l$ & $2 / 5+m$ & $1 / 3+q$ & $l+m+q$ even \\
\hline
\end{tabular}

Here $l, m$ and $q$ are integers.

We recall that the Schwarz's table gives us the cases for which the Galois (and monodromy) Groups are finite (i.e., the identity component of the Galois Group is reduced to the identity element) and is given by fifteen families. These families are given by families $2-15$ of the table above and by the family $(1 / 2+\mathbf{Z}) \times(1 / 2+\mathbf{Z}) \times \mathbf{Q}$. As this last family is already contained in family 1 of the above table, all of the Schwarz's Families are, of course, contained in the above table.

The main result of this note is the following

THEOREM 3. If the Hamiltonian System with Hamiltonian (3.1) is completely integrable with meromorphic first integrals, then each pair $\left(k, \lambda_{i}\right)$ belongs to one of the following list (we assume $k \neq 0$ )

(1) $(k, p+p(p-1) k / 2)$,

(2) (2, arbitrary complex number),

(3) (-2, arbitrary complex number),

(4) $\left(-5, \frac{49}{40}-\frac{1}{40}\left(\frac{10}{3}+10 p\right)^{2}\right)$,

(5) $\left(-5, \frac{49}{40}-\frac{1}{40}(4+10 p)^{2}\right)$,

(6) $\left(-4, \frac{9}{8}-\frac{1}{8}\left(\frac{4}{3}+4 p\right)^{2}\right)$,

(7) $\left(-3, \frac{25}{24}-\frac{1}{24}(2+6 p)^{2}\right)$,

(8) $\left(-3, \frac{25}{24}-\frac{1}{24}\left(\frac{3}{2}+6 p\right)^{2}\right)$,

(9) $\left(-3, \frac{25}{24}-\frac{1}{24}\left(\frac{6}{5}+6 p\right)^{2}\right)$, 
(10) $\left(-3, \frac{25}{24}-\frac{1}{24}\left(\frac{12}{5}+6 p\right)^{2}\right)$,

(11) $\left(3,-\frac{1}{24}+\frac{1}{24}(2+6 p)^{2}\right)$,

(12) $\left(3,-\frac{1}{24}+\frac{1}{24}\left(\frac{3}{2}+6 p\right)^{2}\right)$,

(13) $\left(3,-\frac{1}{24}+\frac{1}{24}\left(\frac{6}{5}+6 p\right)^{2}\right)$,

(14) $\left(3,-\frac{1}{24}+\frac{1}{24}\left(\frac{12}{5}+6 p\right)^{2}\right)$,

(15) $\left(4,-\frac{1}{8}+\frac{1}{8}\left(\frac{4}{3}+4 p\right)^{2}\right)$,

(16) $\left(5,-\frac{9}{40}+\frac{1}{40}\left(\frac{10}{3}+10 p\right)^{2}\right)$,

(17) $\left(5,-\frac{9}{40}+\frac{1}{40}(4+10 p)^{2}\right)$,

(18) $\left(k, \frac{1}{2}\left(\frac{k-1}{k}+p(p+1) k\right)\right)$,

where $p$ is an arbitrary integer.

Proof. The proof follows from our Theorem 1 and from Kimura's Theorem, because if the identity component of the Galois Group is abelian, then, in particular, it is solvable. Case (1) corresponds to case (i) in the Kimura's Theorem and cases (2)-(18) to case (ii) in Kimura's Theorem. In particular, in cases (4)-(18) the Galois Group is finite and the identity component of the Galois Group (of the $A N V E$ and of the $N V E$ ) is trivial.

We recall Yoshida's Theorem. For $n=2$ (only one parameter $\lambda$ appears), let us consider the four regions

(i) $S_{k}=\{\lambda>1,-j(j+1)|k| / 2-j+1>\lambda>-j(j+1)|k| / 2 /+j+1, j \in \mathbf{N}\}$, for $k \leq-3$,

(ii) $S_{-1}=\mathbf{C}-\{-j(j-1) / 2+1, j \in \mathbf{N}\}$,

(iii) $S_{1}=\mathbf{C}-\{j(j-1) / 2+1, j \in \mathbf{N}\}$,

(iv) $S_{k}=\{\lambda<0, j(j-1) k / 2+j<\lambda<j(j+1) k / 2-j, j \in \mathbf{N}\}$, for $k \geq 3$.

Then $([7])$

THEOREM 4. If $\lambda$ is in the region $S_{k}$ then the corresponding Hamiltonian System is not integrable.

Now, it is easy to show that Yoshida's Theorem is a particular case of Theorem 3. We sketch the steps. For the case (1) of the Theorem 3, we see that the parameter $\lambda$ belongs to the complement, in the complex plane, of the Yoshida non-integrability regions $S_{k}, k \in \mathbf{Z}-\{2,-2\}$, because $S_{1}=\mathbf{C}-\{p+p(p-1) / 2\}, S_{-1}=\mathbf{C}-\{p-p(p+$ 1) $/ 2\}$ (with $p \in \mathbf{Z}$ ) and for the rest of the $k$ values $\lambda=p+p(p-1) k / 2$ are precisely the extremities of the open intervals which appear in $S_{k}$. For the cases (3)-(18) we give an indirect argument. As these cases corresponds to a finite Galois (and monodromy) Group and Yoshida's Theorem is based (as Ziglin's Theorem) on the existence of a non-resonant monodromy matrix, but for these cases all the Galois (and monodromy) transformations of the $N V E$ are resonant (we recall that the identity component of the Galois Group is preserved when we obtain the $A N V E$ from the $N V E$, see [4]), then necessarily the values of the Yoshida coefficient $\lambda$ for the cases (3)-(18) are contained in $\mathbf{C}-\cup S_{k}$.

The inquisitive reader can ask why we do not use the last argument (used for cases (3)-(18)) for the case (2). The reason is that, in this case, may be the Galois group has an identity component solvable but not abelian and in Yoshida's (and Ziglin's) Theorem a necessary condition for integrability is the abelianess of the identity com- 
ponent of the Galois Group (in fact, for a two degrees of freedom Hamiltonian System, the Ziglin's Theorem is a consequence of our Theorem 1, see [4]).

\section{Examples.}

EXAMPLE 1. We consider with Yoshida the collinear three body problem with a homogeneous potential

$$
V\left(q_{1}, q_{2}, q_{3}\right)=\left|q_{1}-q_{2}\right|^{k}+\left|q_{1}-q_{3}\right|^{k}+\left|q_{3}-q_{1}\right|^{k}
$$

By a reduction to the center of masses it is transformed to a two degrees of freedom potential

$$
V\left(x_{1}, x_{2}\right)=\left(\sqrt{3} x_{1}+x_{2}\right)^{k}+\left(-\sqrt{3} x_{1}+x_{2}\right)^{k}+\left(2 x_{2}\right)^{k} .
$$

For $k$ arbitrary integer it is possible to obtain an hyperelliptic integral curve with $A N V E$ having Yoshida parameter $\lambda=3(k-1) /\left(1+2^{k-1}\right)$. For $k$ even and positive there exists an additional hyperelliptic integral curve with an $A N V E$ having $\lambda=(k-1) / 3([7])$.

By applying the last theorem we get

Proposition 1. Except for the four cases $k=-2,1,2,4$, the collinear (homogeneous) potential of three particles of degree $k$ is not integrable (we do not consider the trivially integrable case $k=0$ ).

We remark that with respect to Yoshida's results the new feature is the nonintegrability of the system for $k$ odd and greater than or equal to 5 . Furthermore, the four cases $k=-2,1,2$ and 4 are well known integrable systems (see the references in [7]). In this way we close the integrability problem for this family.

ExAmple 2. We will apply Theorem 3 to a family of systems with an arbitrary number of degrees of freedom studied by Umeno ([6]).

In order to avoid the already mentioned arithmetical problems (for $n$ greater than two) related to the non-resonance condition, Umeno introduced the non-resonancedegenerate-condition. In this way he studied the non-integrability of the systems given by the very symmetric $n$-degrees of freedom homogeneous potentials

$$
V=\frac{1}{s} \sum x_{i_{1}}^{s} x_{i_{2}}^{s} \cdots x_{i_{r}}^{s}
$$

where in the summation appear all the possible combinations of $r$ different integers, $i_{1}, i_{2}, \ldots, i_{r}$, with $i_{p}$ equal to $1,2, \ldots, n$. With Umeno we will denote this Hamiltonian System by $(n, r, s)$. We observe that, in any case, $r \leq n$.

For these systems, we have $k=r s$ and it is possible (see [6]) to find a hyperelliptic curve with associated $A N V E$ admitting as Yoshida parameters the values

$$
\lambda_{1}=\lambda_{2}=\cdots=\lambda_{n-1}=s-1-\frac{s(r-1)}{n-1}:=\lambda .
$$

Then, by Theorem 3, we get

Proposition 2. The above Hamiltonian Systems with parameters $(n, r, s)(2 \leq$ $n)$ are not completely integrable, except perhaps for the five cases

(i) $n(s-1)=r s-1$,

(ii) $n(s-2)=r s-2$, 
(iii) $r=1$,

(iv) $r s=2$

(v) $2\left(s-1-\frac{s(r-1)}{n-1}\right)=\frac{s r-1}{s r}$.

Proof. We need to check cases (1), (2) and (11)-(18) of Theorem 3. Case (iv) corresponds to case (2). Now, it is easy to see that $\lambda=s-1-\frac{s(r-1)}{n-1} \leq r s-1$ (this fact is also used in [6]). Hence, it is only necessary to consider the values $p=0,1$ and -1 in the case (1) of Theorem 3 (for other values of $p$ we do not have positive integer values of $n, r$ and $s$ ). These values gives us the conditions (i), (ii), (iii) and (v). This last case corresponds to (18), $p=0$.

Case (11) is not possible, because $r s=3$ implies $r=1, s=3$ or $r=3, s=1$. As $r=1$ appears in (iii), it is only necessary to consider $r=3, s=1$. But the equation $-2 /(n-1)=-1 / 24+(2+6 p)^{2} / 24$ has not integer solutions $n$ and $p$. In a similar way, the cases (12)-(17) are not possible.

We observe that the trivial case (iii) corresponds to a separable potential.

In his paper Umeno proved the non-existence of an additional integral for the following systems:

(a) $(n, r, 1)$ with $3 \leq r$,

(b) $(n, r, 2)$ except for the five cases $(2,2,2),(3,3,2),(26,4,2),(6,6,2),(28,6,2)$, the two families $(2 r-1, r, 2),(r+1, r, 2)(2 \leq r)$ and the trivial family $r=1$.

(c) $(n, r, s)$ with $2<s, \frac{r s-2}{s-2}<n$ and $2 \leq r$.

We remark that condition (c) is incompatible with condition (v) of Proposition 2.

We first notice that $n-1>0$. Then condition (v) implies $2\left(s-1-\frac{s(r-1)}{n-1}\right)=\frac{s r-1}{s r}<1$. Therefore $2(s-1)(n-1)-2 s r+2 s<n-1$ and $2 s(n-r)<3(n-1)$. From condition (c) we get $s>2$ and $r s-2<s n-2 n$. Therefore $2 s(n-r)>4(n-1)$. Finally $3(n-1)>4(n-1), 0>n-1$, and we get a contradiction.

Using similar (simpler...) arguments, starting from Proposition 2 we can prove the non-complete integrability of systems (a), (b) and (c) and, furthermore, we get new non-integrable systems. For instance, the systems of type $(n, r, 2)(2 \leq n)$ are not completely integrable except for either $(n, 1,2)$ or $(2 r-1, r, 2)$. Umeno considers in [6] as an open problem for these systems, with $r=2$, the fact that among the systems in (b) there are some of them which have a non integer difference of Kowalevski exponents, but his criterion leaves open their non-integrability. We have proved above that all these systems are never completely integrable.

We note that the non integrable system with parameters $(2,2,2)$ is the Yang-Mills potential studied by Ziglin in [8].

As a final remark, we think that it is possible to apply Theorem 3 to other interesting systems.

ACKnowledgements. In a first version of this paper (june 1997), case (18) of Theorem 3 was missing. We noticed this fact after that Andrzej J. Maciejewski and Haruo Yoshida signaled us independently counterexamples to our statement. We thank them for their interest in our work and their help. Also we would like to thank Carles Simó, who read a preliminary manuscript and encouraged us to publish it, and Josep M. Peris for interesting discussions.

The first author was partially supported by the Spanish grant DGICYT PB940215, the EC grant ERBCHRXCT940460 and the Catalan grant CIRIT 1996S0GR- 
00105.

\section{REFERENCES}

[1] I. Kaplansky, An Introduction to Differential Algebra, Hermann, 1976.

[2] T. Kimura, On Riemann's equations wich are solvable by quadratures, Funkcialsj Ekvacioj, 12 (1969), pp. 269-281.

[3] J. Martinet and J. P. Ramis, Théorie de Galois différentielle et resommation, in Computer Algebra and Differential Equations, E. Tournier, ed., Academic Press, 1989, pp. 117-214.

[4] J. J. Morales-Ruiz And J. P. Ramis, Galoisian obstructions to integrability of Hamiltonian systems, Methods and Applications of Analysis, 8 (2001), pp. 33-96.

[5] M. F. Singer, An outline of differential Galois theory, in Computer Algebra and Differential Equations, E. Tournier, ed., Academic Press, 1989, pp. 3-57.

[6] K. Umeno, Non-integrable character of Hamiltonian systems with global symmetric coupling, Physica, 82D (1995), pp. 11-35.

[7] H. Yoshida, A criterion for the non-existence of an additional integral in Hamiltonian systems with a homogeneous potential, Physica, 29D (1987), pp. 128-142.

[8] S. L. Ziglin, Branching of solutions and non-existence of first integrals in Hamiltonian mechanics II, Funct. Anal. Appl., 17 (1983), pp. 6-17. 